\title{
Relation priming in established compounds: facilitation?
}

\author{
Thomas L. Spalding • Christina L. Gagné
}

Published online: 20 May 2011

(C) Psychonomic Society, Inc. 2011

\begin{abstract}
Gagné and Spalding (Brain and Language, 90, 478-486, 2004, Journal of Memory and Language, 60, 20 $35,2009)$ have shown that the difficulty of interpreting an established compound (e.g., snowball) can be influenced by recent exposure to a compound with the same modifier and that this influence depends on the relation linking the constituents of the compound. For example, snowball (a ball made of snow) was processed more quickly following snowfort (a fort made of snow; same relation) than following snowshovel (a shovel for snow; different relation). In three experiments, we investigated the basis of this relation-priming effect. The results indicated that the relation-priming effect in established compounds is due to slower processing in the different-relation condition rather than to faster processing in the same-relation condition. These results pose a challenge for most models of compound-word processing.
\end{abstract}

Keywords Compounds · Complex words · Conceptual combination $\cdot$ Noun-noun phrases

Consider an established (familiar) compound such as teacup. A speaker using the compound before it became a word in the language would have needed to combine the concepts tea and cup to express the meaning a cup for tea. Furthermore, people hearing teacup for the first time are likely to be able to understand it if they know the concepts corresponding to the words tea and cup. In either case, the

T. L. Spalding $(\bowtie) \cdot$ C. L. Gagné

Department of Psychology, University of Alberta,

P-217 Biological Sciences Building,

Edmonton, Alberta T6G 2E9, Canada

e-mail: spalding@ualberta.ca speaker or hearer must combine the constituent concepts in order to create a meaning for the compound. For this reason, the conceptual combination process is important in the initial development of compound words and continues to be important for the understanding of compounds that are new to the hearer.

During the conceptual combination process, the interpretation often makes use of a relation to link the concepts, such as noun-FOR-modifier in the example of teacup above. The relation, though not overtly expressed in the compound, has important implications for the meaning of the phrase: a cup for tea is quite different from a cup made of tea or a cup that causes tea or a cup that is caused by tea, and so on. Thus, the interpretation of the compound includes the relation as well as the meanings of the constituent words (see Pelletier, 2003, for a related philosophical argument). Linguists interested in how compounds are interpreted have developed several similar classification schemes for the relations that link the constituents of compounds (e.g., Downing, 1977; Levi, 1978; Warren, 1978).

A number of studies have shown that such relations influence the processing of novel compounds (Devereux, Maguire, Costello, \& Cater 2006; Estes \& Jones, 2006; Gagné, 2001, 2002; Gagné \& Shoben, 1997, 2002; Gagné \& Spalding, 2006; Gagné, Spalding, Figueredo, \& Mullaly 2009; Gagné, Spalding, \& Ji 2005b; Jones, Estes, \& Marsh 2008; Maguire \& Cater, 2004; Maguire, Devereux, Costello, \& Cater 2007; Ramey, 2005; Spalding \& Gagné, 2007, 2008; Spalding, Gagné, Mullaly, \& Ji 2010; Storms \& Wisniewski, 2005). For example, effects of such relations were demonstrated in a relation-priming paradigm using novel compounds by Gagné (2001). Target novel compounds such as mountain bird (LOCATED) were interpreted more quickly following a same-relation prime such as 
mountain stream (LOCATED) than following a differentrelation prime such as mountain magazine (ABOUT).

One important finding in this literature is that relational effects are mostly associated with the modifier when the task requires creating an interpretation. For example, Gagné (2001) found no relational effects associated with the head noun, although they found robust relational effects associated with the modifier (see also Gagné \& Shoben, 1997; Gagné et al., 2005b; Jones et al., 2008; Maguire \& Cater, 2004; Ramey, 2005; Storms \& Wisniewski, 2005). Headbased relational effects have been found when the task requires only the evaluation of a given interpretation (Spalding et al., 2010). In the present article, we focus on the modifier-based effects, because the task of interest is the creation of an interpretation of the compound.

Gagné and Spalding (2004, 2009) demonstrated that relation priming occurs for established as well as for novel compounds. Compounds such as snowball (ball MADE OF snow) were processed more quickly following a prime such as snowfort (fort MADE OF snow) than following a prime such as snowshovel (shovel FOR snow). Gagné and Spalding (2004, 2009) interpreted such relation-priming effects as showing that relational interpretation is common to novel and established compound processing. It is not clear, however, what process gives rise to the relational-priming effect in established compounds. In particular, it is unclear whether the priming effect arises because processing is facilitated in the same-relation condition, because processing is impeded in the different-relation condition, or a combination of both.

The main aim of the present article is to investigate the extents to which the relation-priming effect in established compounds is due to facilitated processing, impeded processing, or a mixture of both, in order to differentiate between various theoretical views of compound processing. We begin by briefly reviewing what mechanisms theories of novel-compound processing have used to account for relation-priming effects in novel compounds, and how they might be extended to established compounds. Theories of established-compound processing have generally not included relational effects, because relational effects for such compounds have only recently been shown. Therefore, we will postpone our discussion of such theories until the General Discussion.

\section{Extending theories of novel compound processing}

Although some theories proposed to account for the processing of novel compounds have not been extended to established compounds (e.g., Estes, 2003; Wisniewski, 1997), we consider here what these theories would predict if extended. It should be noted that these theories as originally conceived might not have assumed that established compounds would be processed in a way similar to novel compounds at all-they might well have assumed that the interpretation of established compounds would consist of retrieval of stored meanings (as do many theories of established-compound processing; see Gagné \& Spalding, 2004, 2006, 2009, for brief discussions). However, this retrieval approach would not account for the relation-priming effects found by Gagné and Spalding (2004, 2009), so we will assume here that some extension of the theories to include established compounds is appropriate.

Estes (2003; Estes \& Jones, 2006) explained relation priming in novel compounds by assuming an independent relation representation (i.e., a relation that is not dependent on or specially linked to either the modifier or head) that is activated by the prime. Thus, when the target item is encountered in the same-relation condition, the required relation is already activated, so performance is speeded. Extending this theory to established compounds straightforwardly suggests that relation priming is due to facilitation.

The schema modification theory (e.g., Murphy, 1988, 1990) and its derivatives (e.g., Wisniewski, 1997) have a slightly more complicated approach to explaining relational priming. They explain priming in novel compounds by assuming that repeating the relation in the target item changes the same slots in the head noun as in the prime. For example, a prime like snowfort changes the material slot of fort, and this change is transmitted to the material slot of ball for the target snowball (i.e., the material slot of ball is predisposed to change due to the material slot of fort having changed). The mechanism that underlies this effect is assumed to result in facilitation with repetition, since Murphy (1990) suggested that the activation level of the slot increases when changed by combination. How this change is transmitted from fort to ball is not specified, however. Extending this mechanism (preactivation of the required slot) to established compounds also predicts that the relational priming in established compounds is due to facilitation when the relational interpretation is repeated.

The RICE (Relational Interpretation Competitive Evaluation; Spalding et al., 2010) theory of compound interpretation is a development and extension of the CARIN (Competition Among Relations In Nominals; Gagné \& Shoben, 1997) theory. According to RICE, the interpretation of a compound is arrived at through a suggestevaluate-elaborate process. Modifiers suggest relations with a strength proportional to the relations' availability for the modifier. The appropriateness of the suggested relations is then evaluated for the modifier and the head of the combination. Finally, once a suggested relation has been selected and evaluated as appropriate, there is an elaboration stage in which the full meaning of the compound is 
developed. Gagné and Shoben (1997) showed that the ease of interpretation of novel compounds depended on the relative frequency with which the required relation had previously been used with the modifier. Recently, Spalding and Gagné (2008) showed that the main determinate of ease of interpretation across items in the Gagné and Shoben (1997) data was the number of "strong but wrong" relations for the modifier. That is, the greater the number of relations that were more frequent (for the modifier) than the relation required for the target compound, the slower participants were to determine that the target compound made sense (see Spalding \& Gagné, 2008, and Spalding et al., 2010, for discussions of the implications of this finding for understanding the process of novel-compound interpretation). In the CARIN theory, relation priming was assumed to result from a change in the activation with which the modifier suggested the repeated relation, relative to the other relations (see, e.g., Gagné, 2001). However, Spalding et al. found that relation priming occurred even when participants were asked only to verify a single relation, suggesting that the relation-priming effect was not due to a relative change among the suggested relations, but to a change in the ease with which the system could settle on the target relation in the evaluation stage. Supporting this interpretation, Spalding et al. found that the number of stronger competitors for the modifier did not affect RTs in the verification task. Thus, in RICE, relational-priming effects are assumed to arise primarily within the evaluation stage of interpretation, rather than in the relation suggestion stage.

RICE's claim that relational-priming effects occur in the evaluation stage suggests that relation priming in established compounds is not likely to be due to facilitation. The meaning of an established compound should be quite strong, even when it has not been primed in any way. Therefore, the effects of relational priming for established compounds might generally be due to the activation of information supporting a competing interpretation in the different-relation condition, rather than to additional activation of information supporting the already dominating meaning in the same-relation condition.

\section{Facilitated and impeded processing of compounds}

Although it might seem intuitively obvious that repeating a relational interpretation should facilitate processing, some existing evidence has suggested that relation-priming effects might result from a mixture of facilitated and impeded processing, and that this mixture might depend on competition among the activated relations. Gagné and Shoben (2002) investigated relation priming in ambiguous novel compounds. Each ambiguous compound had a dominant interpretation (a meaning that most people preferred) and a less preferred interpretation. Relationpriming effects were relatively small for the dominant interpretations, but relatively large for the less preferred interpretations. Similarly, Spalding and Gagné (2007) showed that the degree to which a given relational interpretation was acceptable for a particular ambiguous noun-noun combination depended on the semantic information recently active about the head. The effect of the activated semantic information was strong for the less preferred interpretations, but relatively weak for the dominant interpretations. Gerrig (1989) found that activating the established meaning of a compound interfered with the ability to construct novel meanings. Gagné, Spalding, and Gorrie (2005a) investigated the interpretation of novel and established compounds that were presented following sentences that included either the dominant meaning of the compound or a more innovative meaning. When the preceding interpretation was not the usual meaning of the compound, responses to the conventional meaning of even highly familiar compounds were slowed, and the likelihood that a participant would accept the conventional meaning of the compound as plausible decreased.

Taken together, these findings suggest that when a compound has a meaning that is relatively dominant or established, the cognitive system would not ordinarily have any difficulty ruling out any of the competing meanings in the evaluation stage, so the dominant or established meanings seem to be relatively difficult to facilitate. On the other hand, strongly activating a competing meaning can make it more difficult to settle on that dominant or established meaning. Thus, for dominant or established meanings, and specifically for the effects demonstrated by Gagné and Spalding (2004, 2009), these results suggest that the relational-priming effect might mostly be one of increased difficulty of interpretation in the different-relation condition.

\section{The present experiments}

Although Gagné and Spalding $(2004,2009)$ showed that the same-relation prime condition was fast relative to the different-relation prime condition, such data cannot differentiate facilitated processing in the same-relation condition from impeded processing in the different-relation condition. Differentiating these two possible explanations would require a baseline that simultaneously fulfills two criteria. First, there must not be an explicit relational interpretation in the baseline prime, because any explicit relational interpretation in the prime would have to be either the same as or different from the relation used in the target. Second, the baseline must control for the priming associ- 
ated with the repeat of the first constituent in the prime and target compounds in the same- and different-relation prime conditions.

In the present experiments, the baseline condition had only the first constituent of the target compound as the prime. The other conditions utilized same-relation and different-relation primes. The first-constituent-only prime condition had no relational interpretation (given that it was not a compound) but had the same lexical and orthographic overlap with the target as the same- and different-relation condition primes, and thus fulfilled the two criteria for the appropriate baseline to determine whether relation-priming effects in compounds are due to facilitated processing in the same-relation prime condition or to impeded processing in the different-relation prime condition.

\section{Experiment 1}

In this experiment, participants performed a sense/ nonsense judgment task. The target items (e.g., snowball) were preceded by one of three types of prime: a compound that shared the first constituent and the same relation (snowfort), a compound that shared the first constituent but had a different relation (snowshovel), or the first constituent only (snow). This experiment allowed us to replicate the relation-priming effect shown in Gagné and Spalding $(2004,2009)$ and to determine whether that effect is due to facilitated processing when repeating the relation, impeded processing when using a different relation, or a mixture of both. If the effect is due purely to facilitation, then the same-relation condition would be fast and the different-relation and the first-constituentonly conditions would be slow. If the effect is due purely to impeded processing, then the different-relation condition would be slow and the same-relation and firstconstituent-only conditions would both be fast. If there is a mixture of both, then the same-relation condition should be fast, the different-relation condition should be slow, and the first-constituent-only condition should be in the middle.

\section{Method}

Participants A total of 42 first-year psychology students participated for course credit. Of these participants, 7 were excluded from the study due to self-report of doing a different task, such as deciding whether each constituent was a word, or due to empirical evidence of not performing the task, such as extremely fast (i.e., under 200-ms) response times (RTs). Another 3 were excluded for having accuracy rates less than $80 \%$ on the target items. Thus, we analyzed the data from 32 participants.
Design This experiment used a one-way design with three conditions: same-relation prime, different-relation prime, and the baseline, first-constituent-only prime.

Materials The materials were taken from Gagné and Spalding (2004). A total of 84 compound words were randomly selected from a list of items that had been gathered from the Brown corpus and various online dictionaries. These words were used as target items. For each target item, two prime compounds with the same first constituent were selected, such that one had the same relation as the target and one had a different relation than the target item, using the Levi (1978) taxonomy of relations. The first-constituent-only condition used just the modifier from the target as the prime.

All of the compound words in the experimental items were used in a speeded lexical decision task, along with filler items consisting of pseudocompounds created by combining two words. Across all conditions, the compounds were confirmed as words by over $90 \%$ of the participants. Hence, although these compounds were low frequency, participants clearly believed they were words, rather than novel phrases.

The experimental items were counterbalanced such that participants saw each target item only once, and across all participants each target item was seen an equal number of times in all conditions. The experimental items are presented in Appendix A.

Filler pairs were created such that there were equal numbers of sense and nonsense responses for primes and for targets across all items in the experiment. Examples of each type of filler pair are presented in Table 1. Most nonsense compounds were created from pairs of monomorphemic words (e.g., iceknock, eggprairie, dishsquirrel). The filler items also mirrored the target items in terms of the proportion of items that looked like compounds (i.e., two thirds of the nonsense filler primes were nonsense compounds, and one third were monomorphemic-like nonwords, so that the mere appearance could not be used to generate a decision). For such items (i.e., the filler analogue to the first-constituent-only condition), the primes were repeated in the paired filler target, whether that target was sensible (e.g., bas-basketball, hom-homework) or not (e.g., nugg-nuggbottle, friv-frivtree). Thus, across all items, the nature of the prime did not predict the response to the target. Although some compounds are presented with spaces or hyphens or without spaces, all compound stimuli in these experiments (both sense and nonsense) were presented without any spacing or hyphenation, so that the form of the stimulus would be equivalent for all trials and so that the replication of the relation-priming effect in Gagné and Spalding (2004, 2009) would be exact. Introducing an interconstituent space in a closed compound 
Table 1 Example experimental and filler prime-target pairs for Experiments 1 and 2

\begin{tabular}{llllll}
\hline Pair Type & Response to Prime and Target & Proportion & Relation & Prime & Target \\
\hline Experimental & \multirow{2}{*}{ Sense-sense } & $1 / 3$ & Same & guncase & gunpowder \\
& & $1 / 3$ & Different & gunbarrel & gunpowder \\
& & $1 / 3$ & N/A & gun & gunpowder \\
Filler & \multirow{2}{*}{ Nonsense-nonsense } & $2 / 3$ & N/A & vasespill & vasebrand \\
& & $1 / 3$ & N/A & nugg & nuggbottle \\
Filler & Nonsense-sense & $2 / 3$ & N/A & birthtube & birthmark \\
& & $1 / 3$ & N/A & bas & basketball \\
Filler & Sense-nonsense & $2 / 3$ & N/A & iceberg & iceknock \\
& & $1 / 3$ & N/A & car & carhud \\
\hline
\end{tabular}

disrupts reading of the compound, whereas removing a space from an open compound does not influence compound processing (Juhasz, Inhoff, \& Rayner 2005).

Procedure Each trial began with the word "Ready?" When the participant pressed the space bar, a prime item appeared. The participant indicated whether the item made sense by pressing the " $\mathrm{J}$ " key for sense or the " $\mathrm{F}$ " key for nonsense. After the participant responded, the word "Ready?" appeared. When the space bar was pressed, the target item appeared and the participant made a sense/nonsense judgment. The experimental and filler item pairs were presented in a randomized order for each participant. Nothing in the presentation (other than order) differentiated prime and target items. Prior to viewing the experimental items, participants were familiarized with the task using practice trials with eight pairs of items that did not occur in the experimental trials.

\section{Results and discussion}

RTs on the correct responses to target items were trimmed by removing responses more than 2.5 standard deviations (SDs) greater than the participant's mean RT by condition (3.61\% of the data). Mean RTs are presented by condition in Table 2. The RTs were transformed to minimize skewness and analyzed using a linear mixed-effect (LME) analysis (Baayen, 2004, 2007, 2008; Bates, 2005; Pinheiro \& Bates, 2000), with participants and items as random effects and prime condition as a fixed effect. Both logtransformed and inverse-transformed data were analyzed. In these data sets, the inverse transform was more effective in normalizing the data (as indicated by inspection of the Q-Q plots and frequency histograms), but log transforms are, perhaps, more common and familiar for use with RT data. In all cases, the results of the log- and inverse-transformed data analyses agreed. The $p$ values reported for the tests of the fixed effects (i.e., tests of the slope associated with the predictor variable) are based on the posterior distributions that were obtained using the Monte Carlo Markov chain (MCMC) sampling procedure (Baayen, Davidson, \& Bates 2008). LME analyses have a number of advantages over traditional ANOVAs (see, e.g., Baayen, 2004, 2008; Baayen et al., 2008). Among other things, LME allows the simultaneous treatment of items and participants as random effects, it allows the analysis of accuracy as a binomial variable (thus avoiding problems with the analysis of accuracies as proportions), it allows the inclusion of categorical and noncategorical predictors in the same model, and it does not require aggregation of the data.

For the inverse-transformed RT data, analysis of the coefficients indicated that responses in the different-relation prime condition were slower than responses in the samerelation prime condition, $t=2.5, p=.01$, and in the firstconstituent-only prime condition, $t=3.9, p=.001$. However, the same-relation and the first-constituent-only prime conditions did not differ, $t=1.4, p=.16$. For the logtransformed RT data, responses in the different-relation prime condition were slower than responses in the samerelation prime condition, $t=2.4, p=.03$, and in the firstconstituent-only prime condition, $t=3.4, p=.001$. The same-relation and first-constituent-only conditions did not differ, $t=1.0, p=.30$.

LME analysis of response accuracy (which was treated as a binomial variable and for which untrimmed data were used in all experiments) showed no significant differences among the same-relation, different-relation, and first-constituent-only conditions (proportions correct were .97, .96, and .97,

Table 2 Mean RTs to target items (standard errors in parentheses) in Experiments 1 and 2, including two different data trims for Experiment 2

\begin{tabular}{llll}
\hline Prime & Exp. 1 & $\begin{array}{l}\text { Exp. 2, } \\
\text { RT }<2.5 S D\end{array}$ & $\begin{array}{l}\text { Exp. 2, } \\
\text { RT < 3,000 ms }\end{array}$ \\
\hline 1st constituent only & $684(16)$ & $793(19)$ & $776(14)$ \\
Same relation & $691(17)$ & $772(12)$ & $772(12)$ \\
Different relation & $709(20)$ & $808(20)$ & $792(15)$ \\
\hline
\end{tabular}


respectively). A model including prime condition did not lead to a better fit to the data than a model that did not include prime condition, $\chi^{2}(2)=2.2, p=.34$.

Responses to the target in the different-relation prime condition were slower than in the other conditions. This experiment replicated the difference between the same- and different-relation prime conditions found by Gagné and Spalding $(2004,2009)$. The pattern of RT data was consistent with impeded processing in the differentrelation prime condition and presented no evidence of facilitation due to repetition of the relation.

Posttest The failure to find facilitation for the same-relation condition relative to the first-constituent-only condition could be due to a poor match between the relations in the primes and targets; note, however, that this explanation would not explain why the different-relation condition was slower than the first-constituent-only condition. To examine whether (as intended) the same-relation primes used the same relations as the targets and the different-relation primes used different relations than the targets, we performed a posttest in which 46 participants were presented with sets of items and asked to verify the intended relational definitions. For example, participants would see snowball = a ball made of snow, and would be asked whether they agreed with the definition. Each participant saw a subset of the items, such that no modifier was repeated, in order to avoid priming among the items to be verified. Overall, participants agreed with the intended interpretation $93 \%$ of the time for target items, $91 \%$ of the time for the same-relation primes, and $91 \%$ of the time for the different-relation primes. The participants were also presented with filler items that were given incorrect paraphrases. The relational paraphrases for the fillers were rejected $89 \%$ of the time; thus, the high levels of agreement for the experimental items were not due to a bias to agree with the presented paraphrases. In general, the intended relations for the experimental items do appear to be the relations likely to have been used by participants in the experiments, and in particular, the failure to find facilitation in the same-relation condition was not due to mismatching relations between the primes and targets in that condition.

\section{Experiment 2}

An alternative explanation for the results of Experiment 1 is that the first-constituent-only, same-relation, and differentrelation conditions might have differed in the semantic similarity or association between the primes and targets. Consequently, part or all of the relational-priming effects in Experiment 1 could be due to semantic similarity or association between the primes and targets. For example, it could be the case that the target items are highly frequent members of the class of items that use the modifier (e.g., snowball might be highly frequent among the set of snowthings), so that perhaps the modifier, even when presented by itself, activates many items that are more semantically similar to or associated with the target item, and the samerelation primes also activate such items. Similarly, perhaps the same-relation prime and the modifier could be more semantically or associatively similar to the target than the different-relation prime. Hence, the pattern of priming results obtained in Experiment 1 might be due to this factor, rather than to the role of relations. Thus, in the present experiment we replicate Experiment 1, but using materials that allow us to statistically control the semantic similarity and association between the primes and targets (see Baayen, 2004, for arguments in favor of using statistical, rather than experimental, control) by entering measures of semantic similarity between the primes and targets as covariates in all analyses.

\section{Method}

Participants A total of 96 first-year psychology students participated for course credit. Nine of these participants were removed from all analyses due to having low accuracy rates (less than $75 \%$ correct to the target items). Another 4, who reported learning a language other than English at a young age and who had unusual RT patterns, were also removed from all analyses. Participants were prescreened to be native monolingual English speakers. However, this prescreening relied on self-report. The age of acquisition reported by the latter 4 excluded participants at the experiment session suggests that they were almost certainly fully bilingual. In addition, these participants had one condition (the first-constituent-only condition) in which their mean RTs exceeded each of their other condition means by more than $30 \%$ (an average difference of $296 \mathrm{~ms}$ from the closest other condition mean). Given the total mean RTs of approximately $800 \mathrm{~ms}$, such condition means are clear outliers. Indeed, no other participant in the experiment had any condition mean that deviated from the closest other condition mean by $30 \%$, suggesting that these participants were doing something quite different from the other participants. The data from the remaining 83 participants were included in the analyses.

Materials The materials were mainly selected from Experiment 1 , with additional items drawn from online dictionaries and text corpora. The 42 experimental prime-target pairs are presented in Appendix B. Latent semantic analysis (LSA; see Landauer, 2002; Landauer \& Dumais, 1997) similarities for all the prime-target pairs used in this 
experiment were entered into the analyses as control variables. Two LSA measures were used: the LSA similarity of the prime to the target (as a whole word) and the LSA similarity of the prime to the target's head only. The mean LSA values are presented in Table 3. It was not possible to select a sufficient number of items for which all conditions had equal values for each of the LSA measures. Therefore, the LSA measures were left free to vary, rather than being experimentally manipulated (there is also reason to believe that experimentally matching the LSA measures, even if it were possible, would have induced severe sampling bias; see Baayen, 2004). For both LSA measures, the same- and different-relation prime conditions did not differ $(p s>.1)$. However, the first-constituent-only prime had significantly higher similarity to the target than did either the same- or the different-relation prime for both measures (all $p \mathrm{~s}<.001$ ). We statistically controlled for a possible effect of LSA similarity on target RT by entering the LSA measures into the LME analyses, so that the tests of the effects of condition would not be confounded with any effect of the semantic similarities.

Procedure The procedure was identical to that for Experiment 1.

\section{Results and discussion}

RTs on the correct responses to target items were trimmed by removing responses more than $2.5 S D$ s greater than the participant's mean RT for the condition (3.97\% of the data). Mean RTs are presented by condition in Table 2. The RTs were transformed to minimize skewness (again, both log and inverse transforms were used) and were submitted to LME analysis with participants and items as random effects. Two control variables were also entered: LSA similarity between the prime and the target as a whole word and LSA similarity between the prime and the target's head only. Finally, condition was entered as a fixed effect and the predictor of interest.

We included the LSA measures as covariates in the models to ensure that any differences between the prime conditions in the analyses could not be due to the differences in LSA similarities. In fact, neither measure of LSA

Table 3 Mean LSA similarities between primes and targets (standard errors in parentheses) in Experiment 2

\begin{tabular}{lll}
\hline Prime & Target (Whole Word) & Target (Head Only) \\
\hline 1st constituent only & $.44(.30)$ & $.30(.21)$ \\
Same relation & $.26(.29)$ & $.17(.12)$ \\
Different relation & $.32(.28)$ & $.14(.11)$ \\
\hline
\end{tabular}

similarity was a significant predictor of RT when entered in an LME model as the only predictor of interest (i.e., without the condition factor). Neither of the LSA similarity measures reached statistical significance when condition was added to the model, either. In general, then, semantic similarity had relatively little effect on the target RTs. This should not be surprising, because the overall levels of semantic similarity were relatively low and all target items had lexical and orthographic overlap with their primes, and so were relatively strongly primed, regardless of the level of semantic similarity. In short, it is unlikely that the LSA differences between conditions affected RTs. Nevertheless, in all of the reported models, we included the LSA measures as covariates so that any potential small effect of semantic similarity was statistically controlled, in order to ensure that the effect of prime condition was not confounded with semantic similarity.

For the inverse-transformed RT data, tests of the coefficients indicated that the different-relation condition differed from the same-relation condition, $t=2.2, p<.05$. The different-relation condition also differed from the firstconstituent-only condition, $t=2.9, p<.05$. The samerelation and first-constituent-only conditions did not differ, $t=1.0, p=.26$. For the log-transformed RT data, tests of the coefficients indicated that the different-relation condition differed from the same-relation condition, $t=2.6, p<.05$. The different-relation condition also differed from the first-constituent-only condition, $t=2.1, p<.05$. The same-relation and first-constituent-only conditions did not differ, $t=0.2, p=.92$.

Although these results are identical to those of Experiment 1, two issues might be somewhat troubling and required further investigation. First, inspection of the means suggested that there could be some facilitation in the same-relation prime condition, although the analyses did not find any evidence of such facilitation. Second, inspection of the raw data and of the Q-Q plots and histograms of the transformed data (for both $\log$ and inverse transforms) showed clear outliers remaining, despite the 2.5-SD cutoff used in the previous analyses. These outliers fell between 3,000 and 7,000 ms, which is clearly outside the norm for sense/nonsense decisions to compounds, and they might have masked an effect of facilitation for the same-relation condition by greatly increasing the error term in the analyses, or they might have distorted the means (or both, of course). Thus, we applied an additional cutoff at 3,000 ms and reanalyzed the data. This cutoff removed 12 additional data points (approximately an additional $0.4 \%$ of the data). The means and standard deviations with this additional cutoff are presented in Table 2. For the inverse-transformed RT data, tests of the coefficients indicated that the different-relation condition differed from the same-relation condition, $t=2.1$, 
$p<.05$. The different-relation condition also differed from the first-constituent-only condition, $t=3.0, p<.05$. The same-relation and first-constituent-only conditions did not differ, $t=1.2, p=.2$. For the log-transformed RT data, tests of the coefficients indicated that the different-relation condition differed from the same-relation condition, $t=2.3$, $p<.05$. The different-relation condition also differed from the first-constituent-only condition, $t=2.3, p<.05$. The same-relation and first-constituent-only conditions did not differ, $t=0.3, p=.72$. The outcomes of the analyses with the data additionally trimmed at $3,000 \mathrm{~ms}$ are the same as the previous analyses. Note, however, that the means no longer suggest any facilitation in the same-relation prime condition.

LME analysis of response accuracy showed no significant differences among the same-relation, different-relation, and first-constituent-only conditions (proportions correct equaled .95, .95, and .94, respectively). A model including prime condition did not lead to a better fit to the data than did a model that did not include prime condition, $\chi^{2}(2)=1.6, p=.45$.

To summarize, even when the LSA measures were included in the model as covariates, the analyses indicated that responses in the different-relation condition were slower than in the same-relation and first-constituent-only conditions, which did not differ from each other. Nonetheless, given the differences between the first-constituent-only condition and the other two conditions in terms of the LSA ratings for primes and targets, and the importance of the first-constituent-only condition as the baseline in the present studies, it was worthwhile to consider whether the pattern of effects could be accounted for by a combination of semantic priming and facilitatory relation priming. In particular, could the first-constituent-only condition be fast due to semantic priming and the same-relation condition be fast due to facilitatory relation priming? In short, no.

If the data were due to a mixture of types of priming, three things should have happened that did not. First, and most critically, the LSA variables should have been significant predictors of RTs; our analyses indicated that the semantic similarity of primes and targets had little effect on target RTs, even when entered as the only predictors in the model. As we discussed above, this is not surprising, given the nature of the materials and task, but it does make it very unlikely that the results could be due to the hypothetical mix of semantic priming and facilitatory relation priming.

Second, the LME models should have indicated a difference between the first-constituent-only and samerelation conditions, in terms of the condition factor (assuming, for the sake of argument, that the LSA ratings predicted some of the RT data). The LME analysis estimates two coefficients to account for a factor with three levels and uses one of the levels as a baseline against which the other levels are adjusted by the coefficients. For example, the LME analysis requires one coefficient to account for the difference between the first-constituent-only condition and the different-relation condition, and one to account for the difference between the same-relation condition and the different-relation condition (i.e., for this example comparison, the different-relation condition is the baseline to estimate the effects of the other two conditions). Because the LSA measures would predict that the samerelation condition should be slow relative to the firstconstituent-only condition, the coefficient for the condition factor would have to bring the predicted RT in the samerelation condition down to the fast level of the firstconstituent-only condition. Thus, we would expect a significant coefficient for the difference between the firstconstituent-only and the same-relation conditions. In no analysis, however, did we find a significant coefficient for the difference between the first-constituent-only and samerelation conditions.

Finally, if the data resulted from a mixture of semantic priming and facilitatory relation priming, but the role of the semantic priming was not robust enough to lead to significant effects in the LME analyses, one would expect, at the very least, that the estimated coefficients for the same-relation condition would be numerically larger in absolute value than those for the first-constituent-only condition (when the different-relation condition is used as the baseline in the LME analysis). However, using the best trimmed data sets, and considering both the analyses with the log- and the inverse-transformed RTs, the estimated coefficients are numerically larger in absolute value for the first-constituent-only condition than for the same-relation condition, the opposite of the prediction from the hypothesized mix. Hence, there is not even a hint in the analyses that the effects could be due to a mix of semantic priming and facilitatory relation priming.

In summary, there was a clear relation-priming effect in this experiment, even with the semantic and associative similarity between the primes and targets statistically controlled. As in Experiment 1, the relation-priming effect appeared to be due to impeded processing in the differentrelation prime condition. Once again, there was no evidence of facilitation in the same-relation prime condition.

\section{Experiment 3}

Using LSA ratings to statistically control for semantic similarity and association between the primes and targets in Experiment 2 allowed us to rule out semantic similarity or association confounds as explanations for the priming pattern that we observed. However, to fully determine that 
the priming pattern was due to impeded processing in the different-relation prime condition, we must also rule out the possibility that the first constituent, when presented by itself, automatically activated the relation used by the target item, thereby causing the first-constituent-only and samerelation prime conditions to be identical and fast because in both conditions the target relation had been activated. If this were the case, then the different-relation condition would be slow simply due to a failure of this automatic activation of the relation. This situation might arise if, for example, the target items in the previous experiments happened to make use of the most likely relations for the first constituents, and if the first constituent when presented by itself activated only or mostly that relation. In short, we needed to rule out the possibility that the first constituent by itself was more relationally (as opposed to semantically or associatively) similar to the same-relation prime than to the different-relation prime.

We did so by using the same- and different-relation prime items from the previous experiment as target items in the present experiment (we will continue to call them the same- and different-relation items for ease of exposition, although this refers only to their role in the previous experiment). These target items (e.g., lamppost and lamplight) were preceded by either the first constituent (e.g., lamp) or a frequency- and length-matched control (e.g., loan). The repeat of the first constituent in the target items should lead to priming for both the same- and different-relation targets due to lexical facilitation or, more generally, to repetition priming. Critically, if the first constituent by itself activated the target item's relation in the previous experiments, then there should be a significant interaction in the present experiment, such that the presentation of the first constituent by itself would lead to significantly more priming for the samerelation target than for the different-relation target.

\section{Method}

Participants A total of 42 first-year psychology students participated for course credit. Of these, 7 had low accuracies (less than $60 \%$ correct in any condition) and were removed from all analyses. The remaining 35 participants were included in the analyses.

Design We used a $2 \times 2$ within-participants design, with targets that had been the same- or different-relation primes in Experiment 2, and primes that were the first constituent of the target or a matched control.

Materials Forty sets of experimental items were selected from Experiment 2 (two sets of items were removed in order to have equal numbers of items in each condition). In addition to these items, monomorphemic control primes were matched to the first-constituent primes in frequency and length. Filler items were adopted from Experiment 2, with additional monomorphemic-like nonwords to equate both the numbers of "yes" and "no" responses and the orthographic overlap between primes and targets in all conditions. Due to the inclusion of these fillers, the correct response to the target was never predictable from the response to the prime.

Procedure The procedure was identical to that of Experiments 1 and 2.

Results and discussion

RTs on the correct responses to target items were trimmed by removing responses greater than $2.5 \mathrm{SDs}$ from the participant's mean RT for the condition $(0.83 \%$ of the data) and then transformed (both log and inverse transforms were used). Means and standard errors are presented in Table 4. Correct RTs to experimental items were analyzed using LME, with participants and items entered as random effects. Target (same vs. different relation) and prime (first constituent vs. control) were entered as fixed effects and the predictors of interest.

Inspection of the means suggests strong effects of prime and of target. In terms of the critical interaction, however, there appears to be little difference. In fact, the priming effect is numerically larger for the different-relation target than for the same-relation target, the opposite of the pattern predicted by the hypothesis that the first constituent by itself activated the relation required by the target in the previous experiments. The analyses are consistent with this description. LME analysis of the inverse-transformed RTs indicated effects of prime, $t=4.7, p<.01$, and target, $t=2.8, p<.01$, but no interaction, $t=1.5, p=.14$. LME analysis of the log-transformed RTs indicated effects of prime, $t=4.5, p<.001$, and target, $t=2.6, p=.01$, but no interaction, $t=-1.4, p=.17$.

Mean accuracies are presented in Table 5. LME analysis of response accuracy showed an effect of prime (first constituent $=89 \%$ vs. control $=83 \%), z=-4.0, p<.001$,

Table 4 Mean RTs to target items (standard errors in parentheses) in Experiment 3

\begin{tabular}{lll}
\hline & $\begin{array}{l}\text { Same-Relation } \\
\text { Target }\end{array}$ & $\begin{array}{l}\text { Different-Relation } \\
\text { Target }\end{array}$ \\
\hline First-constituent prime & $972(35)$ & $898(30)$ \\
Control prime & $1,048(38)$ & $1,027(38)$ \\
\hline
\end{tabular}


Table 5 Mean percent accuracies to target items (standard errors in parentheses) in Experiment 3

\begin{tabular}{lll}
\hline & $\begin{array}{l}\text { Same-Relation } \\
\text { Target }\end{array}$ & $\begin{array}{l}\text { Different-Relation } \\
\text { Target }\end{array}$ \\
\hline First-constituent prime & $88(1)$ & $90(1)$ \\
Control prime & $84(2)$ & $82(2)$ \\
\hline
\end{tabular}

but no effect of target (both conditions $=86 \%$ ), $z<1$, and no interaction, $z=1.1, p=.29$. Again, the interaction was not significant, and the pattern of means was numerically opposite to that predicted by the claim that the first constituent by itself activated the relation required by the target in the previous experiments.

Presentation of the first constituent equally primed both the same- and different-relation items, relative to the matched control prime. There was no hint of increased priming for the same-relation targets, as compared to the different-relation targets. Hence, the pattern of results obtained in Experiments 1 and 2 cannot be due to the first constituent, when presented by itself, differentially activating the relation required by the target items.

\section{General discussion}

In Experiments 1 and 2, the different-relation primes led to slower responses to the targets than did the samerelation primes. These experiments provide replications of the effect of relational priming on compound processing shown by Gagné and Spalding (2004, 2009). In addition, the different-relation primes also led to slower processing of the target than did the first-constituent-only primes. Thus, it appears that encountering a target item using a different relation than the prime impedes the interpretation of the target. Importantly, the same-relation primes did not differ from the first-constituent-only primes; no analysis in any of our experiments suggested facilitation due to repeating the relation. This pattern is consistent with the idea that the relation-priming effect in established compounds is due to impeded processing when the prime relation is different from the target relation, rather than to facilitation when the relation is repeated. In addition, we have ruled out alternative explanations based on a failure to match relations between the same-relation primes and targets, or a confound of semantic or associative similarity between the primes and targets, or, finally, the automatic activation of the required target relation by the first constituent presented by itself. In the rest of this discussion, we will examine how theories that concern processing of either novel or established compounds might explain these results.
Processing novel compounds

As discussed in the introduction, the schema modification theory (Murphy, 1988, 1990) and its derivatives (e.g., Wisniewski, 1997) account for relation priming in novel compounds by facilitation of the changed value of the slot when the relational interpretation is repeated from the prime to the target compound. Similarly, Estes's (2003; Estes \& Jones, 2006) independent-relation representation theory also assumes facilitation of the repeated relation. According to these theoretical approaches, the first-constituent-only condition would not activate any particular relation or slot, so both it and the different-relation condition should be slow as compared to the same-relation condition. Thus, these theories (if directly extended to established compounds) would fail to account for either the impededprocessing effect found in the different-relation condition or the lack of facilitation in the same-relation condition. Accounting for the impeded-processing effects in the present experiments would require adding a new mechanism to the theories. Furthermore, the results of Gerrig (1989), Gagné and Shoben (2002), Gagné et al., (2005a), and Spalding and Gagné (2007) suggest that the degree to which processing is facilitated or impeded depends on whether the meaning is established (or dominant, for novel compounds). Thus, these theories would also require a mechanism to control the degree to which processing is facilitated or impeded based on the extent to which a meaning is established.

The RICE theory (Spalding et al., 2010), on the other hand, directly incorporates competition among relational interpretations for novel compounds. Importantly for the present purposes, one major determinant of the time required to interpret a given novel compound is the need, in the evaluation stage, to rule out relations suggested by the modifier. When this theory is applied to established compounds, the evaluation and elaboration stages should usually be relatively fast for the canonical meaning, due to the person's experience with that meaning. That is, the same-relation condition is unlikely to improve performance, because whatever competing relations are usually suggested by the modifier should be easily ruled out in the elaboration phase, due to the person's experience with the established meaning of the compound. However, having recently used a different relational interpretation should cause difficulty in settling on the established meaning. In short, the different-relation prime increases the availability of information supporting an interpretation that must be ruled out in order to settle on the canonical meaning of the target compound. This makes it harder to rule out that interpretation and settle on the established meaning of the compound in the evaluation stage, and thus slows down the processing of the target (this effect is very much like 
that reported by Gagné et al., 2005a, in which the presentation of an innovative meaning made participants less likely to accept the established meaning of a compound).

The effect of relation priming in the present experiments is, in a sense, similar to the effect of the "strong but wrong" relations in the interpretation of novel compounds, as discussed in the introduction (see also Spalding \& Gagné, 2008; Spalding et al., 2010). In particular, what appears to be causing the relationalpriming effect in compounds is difficulty ruling out the unwanted interpretation when that interpretation has been recently used (and, therefore, is highly available). Similarly, in unprimed novel compounds, much of what determines ease of processing is ruling out unwanted relations suggested by the modifier. Experience with the established compound probably makes it easier to settle on the required interpretation, as compared to novel compounds (and this, in turn, is directly analogous to the effect of the relative dominance of interpretations for novel compounds, as shown in Gagné \& Shoben, 2002, and Spalding \& Gagné, 2007).

Thus, according to the RICE theory, the extent to which processing is facilitated or impeded depends on the degree to which the relational interpretations are easy to rule out in the evaluation stage, and therefore the theory treats novel and established compounds in essentially similar ways, although experience with more established compounds is assumed to make it easier to settle on the canonical meaning. We should note that, although the effects in the present experiments primarily reveal impeded processing, if we had used ambiguous novel compounds with very-low-dominance meanings, we would likely have seen some facilitation (see, e.g., our discussion of Gagné et al., 2005a, and Spalding \& Gagné, 2007, in the introduction). Established compounds, of course, do not have low-dominance meanings. Indeed, "low-dominance" meanings for established compounds would in fact be new or so-called innovative meanings; research by Gerrig (1989) and by Gagné et al., (2005a) has suggested that such meanings can be facilitated by creating supportive contexts.

\section{Processing established compounds}

Most theories of established-compound processing have not considered effects of the relation between the first and second constituents, because until recently there was no evidence that the relational structure of established compounds affected their processing. In particular, most compound-processing theories have assumed that established compound interpretations are accessed from the mental lexicon (see Gagné, 2009; Gagné \& Spalding,
2004, 2006, 2009; Gagne et al., 2009, for brief discussions). We will consider a few ways in which relational competition might be included in compound theories and evaluate how well such theories might account for the present data.

One might suggest that any theory of complex word processing would account for the present data, due to the fact that word meanings are stored in the mental lexicon, and retrieval is faster than construction, so that the compound words could not be facilitated in the samerelation condition. In the different-relation condition, perhaps the wrong meaning might be activated enough to interfere with the usually fast retrieval of the intended interpretation. This view is somewhat similar to our view, but whereas in our view the competition that accounts for the effect is within the interpretation construction process, in this view the competition is only in retrieval of existing interpretations. This retrieval view seems unsatisfactory, however, because there is presumably no preexisting representation of the wrong meaning of the target compound in the mental lexicon to be activated by the differentrelation prime, and so there is no wrong meaning in the mental lexicon to compete with the canonical meaning for retrieval. Therefore, there is no pure retrieval account of the present data.

One could assume a meaning construction process that runs more or less automatically and concurrently with retrieval. Even most decomposition theories of compound processing, however, only assume that there is a route that activates the stored representation through the constituents, which is not enough to account for the present data. Also, such theories generally assume that the routes are independent and race against each other (see, e.g., Schreuder \& Baayen, 1995, 1997), rather than assuming that they directly compete with each other. Such a view would tend to lead to the prediction that relationalpriming effects should only be obtained for low-frequency compounds, as higher-frequency compounds could, presumably, be retrieved far more quickly than they could be constructed. Gagné and Spalding (2009), however, found that the relational-priming effect did not interact with compound frequency, which suggests that an interpretation construction process is operating even for high-frequency compounds.

In any case, this retrieval-plus-construction view must assume that the different-relation prime leads the construction process to create a meaning (the wrong one) nearly as quickly as the retrieval process can access the usual meaning. However, construction of the wrong meaning would encounter strong competition from construction of the correct meaning. Presumably, then, primed construction of the correct meaning, which would be suffering relatively little competition from the wrong meaning, should be even 
faster than retrieval. This in turn would predict facilitation in the same-relation condition. In short, it is not clear that a retrieval-plus-construction process can provide a full account of the present data.

One might instead expand compound-processing theories that include decomposition of the compound into its constituents (e.g., Libben, 1998; Libben, Gibson, Yoon, \& Sandra 2003; Zwitserlood, 1994) by assuming that compounds are stored in the mental lexicon in such a way that compounds that share first constituents and relations are more likely to benefit from the spread of activation from the prime (see Gagné \& Spalding, 2006, for a brief discussion). For example, processing the prime snowfort might activate other compounds that include snow as a modifier and that use the MADE OF relation, as compared to those that use the FOR relation. Even with this extension, however, such theories would most naturally predict a facilitation effect, because the same-relation prime would differentially activate stored compounds with the same relation as the target, as compared to the compounds activated by the first constituent alone. Experiment 3 shows that the first constituent by itself appears to activate compounds without regard to the relation that they are based on, so that this kind of activation view should certainly predict facilitation for the same-relation condition.

Some existing psycholinguistic theories that already include competition might provide insight into how relational structures and competition could be integrated in psycholinguistic theories. For example, Myers (2007) presented a proposal that takes both relational information and competition into account. In particular, Myers proposed that relations could be considered as covert f-morphemes attached to the first constituents in the representations of compounds. The f-morphemes are mutually exclusive; only one can be attached to the first constituent at a particular time, so that the f-morphemes would compete and inhibit each other (e.g., a prime such as snowshovel would be represented as snow-FOR-shovel, and the interpretation of a target such as snowball would be slowed due to the fact that the covert f-morpheme FOR would have to be replaced on snow with the covert f-morpheme MADEOF). Similarly, Marslen-Wilson, Tyler, Waksler, and Older (1994) found that two derivationally complex words that shared a root would not prime each other, even though each derivation would be primed by the root and the root would be primed by each derivation (e.g., confession would not prime confessor, even though both confession and confessor prime, and are primed by, confess). Marslen-Wilson et al. posited that the complex words are represented in a decomposed form, but that there are inhibitory links between the suffixes, or between the links from root to suffix. Their reasoning was that when the root is activated, but needs to be suffixed, the suffixes are then necessarily mutually exclusive (i.e., they cannot both be added to the same root in the same position at the same time), and therefore some inhibitory links between them are necessary, so that both are not simultaneously suffixed to the root.

Two points suggest that these approaches are not sufficient to account for the present results, however. First, it is unclear how such approaches could account for the lack of facilitation in the same-relation prime condition, relative to the first-constituent-only prime condition. For example, although slower responses might well result from the processing to replace the incorrect f-morpheme in the different-relation prime condition, it certainly seems that there should be some processing advantage to having the correct f-morpheme already in place, as in the samerelation prime condition, as compared to having to add the f-morpheme, as in the first-constituent-only prime condition (the results of Experiment 3 rule out the possibility that the target's f-morpheme is already in place when the first constituent is presented alone). Second, both proposals suggest competition for a position linked to a particular word (i.e., the f-morpheme or derivational suffix has to be placed onto a particular word). However, Gagné (2002) found that relation priming occurs when the first constituents are semantically similar, rather than lexically identical (e.g., scholar paper primes student vote more than scholar table). Gagné (2002) takes this as evidence that relational information is represented at the conceptual level, rather than at the lexical level. On Myers's (2007) view, such a relational-priming effect should not occur with first constituents that are only semantically related, because the f-morphemes are not being attached to the same word, and there should thus be no competition. The same would be true, mutatis mutandis, for the extension of the idea of derivational suffixing to compounding.

\section{Conclusion}

We have demonstrated that relation-priming effects for established compounds are primarily due to impeded processing when the relation changes from prime to target rather than to facilitation of a repeated relation. This finding is consistent with an application of the RICE theory of novel-compound interpretation (Spalding et al., 2010) to the processing of established compounds. In particular, the processing of compounds is affected by the competition between relational interpretations, such that activating an interpretation other than the established interpretation (as in the different-relation prime condition in the present experiments) increases the difficulty in selecting the established relational interpretation of the compound.

Author Note This research was supported in part by NSERC Discovery Grant 250028 to the first author and NSERC Discovery Grant 203054 to the second author. 
Appendix A

Table 6 Stimuli used in Experiment 1

\begin{tabular}{lll}
\hline Target & Same Different & $\begin{array}{l}\text { 1st Constituent } \\
\text { Only }\end{array}$
\end{tabular}

\begin{tabular}{|c|c|c|c|}
\hline carport & carwash & carride & car \\
\hline paintbrush & paintbox & paintball & paint \\
\hline raincoat & rainboots & rainwater & rain \\
\hline worktable & workstation & workpeople & work \\
\hline wineglass & winecellar & winedrunk & wine \\
\hline doghouse & dogcollar & dogtrot & $\operatorname{dog}$ \\
\hline milkman & milkmaid & milkshake & milk \\
\hline storyteller & storywriter & storybook & story \\
\hline riverboat & riverraft & riverwater & river \\
\hline farmboy & farmhand & farmland & farm \\
\hline lampshade & lamppost & lamplight & lamp \\
\hline homebrew & homebaking & homeland & home \\
\hline grapevine & grapetree & grapewine & grape \\
\hline sheepskin & sheepwool & sheepshears & sheep \\
\hline treehouse & treefrog & treebranch & tree \\
\hline applesauce & applecake & appletree & apple \\
\hline weekday & weeknight & weekend & week \\
\hline sailboat & sailboard & sailyard & sail \\
\hline eyelash & eyelid & eyeglass & eye \\
\hline waterfall & waterdrop & watercolour & water \\
\hline goldfish & goldleaf & goldmine & gold \\
\hline paperplate & papercup & paperboy & paper \\
\hline notebook & notepad & notetaker & note \\
\hline cheesecake & cheesebread & cheeseknife & cheese \\
\hline snowball & snowfort & snowshovel & snow \\
\hline pincushion & pinholder & pinhole & pin \\
\hline gunman & gunowner & gunfight & gun \\
\hline desklamp & deskorganizer & desktop & desk \\
\hline windowpane & windowledge & windowshop & window \\
\hline doorknob & doorbell & doorman & door \\
\hline potholder & potlid & pothole & pot \\
\hline newspaper & newsletter & newsroom & news \\
\hline bankbook & bankloan & bankrobber & bank \\
\hline teabag & teapot & teacake & tea \\
\hline tinfoil & tincan & tinpress & tin \\
\hline bookcase & bookcover & bookhoard & book \\
\hline sharkskin & sharkcartilage & sharkbite & shark \\
\hline gingerbread & gingertea & gingerplant & ginger \\
\hline fireman & firetruck & firefly & fire \\
\hline footwear & footstool & footprint & foot \\
\hline whalebone & whaleblubber & whaletrade & whale \\
\hline daylight & daytime & daybreak & day \\
\hline honeybee & honeygland & honeycomb & honey \\
\hline earring & eardrops & earhole & ear \\
\hline deathbed & deathscene & deathbell & death \\
\hline
\end{tabular}

Table 6 (continued)

Target Same

Different

1st Constituent

Only

\begin{tabular}{|c|c|c|c|}
\hline necktie & necklace & neckpain & neck \\
\hline breadboard & breadbasket & breadcrumb & bread \\
\hline stonemason & stonecutter & stonework & stone \\
\hline bearskin & bearpaw & bearsuit & bear \\
\hline handbag & handcream & handbrake & hand \\
\hline horseback & horsetail & horseshoe & horse \\
\hline hairbrush & hairclip & haircloth & hair \\
\hline vineyard & vinegarden & vinecutter & vine \\
\hline backbone & backpain & backtalk & back \\
\hline sandstone & sandcastle & sandbox & sand \\
\hline spacesuit & spaceship & spaceman & space \\
\hline sunlight & sunbeam & sundeck & sun \\
\hline toybox & toyshelf & toysoldier & toy \\
\hline tombstone & tombsite & tombmaker & tomb \\
\hline marketplace & marketsquare & marketfresh & market \\
\hline shoelace & shoeclasp & shoehorn & shoe \\
\hline cannonball & cannonfuse & cannonsmoke & cannon \\
\hline mousetrap & mousehole & mousetail & mouse \\
\hline tablecloth & tablespoon & tableleg & table \\
\hline seaweed & seawater & seabreeze & sea \\
\hline wintertime & winterchill & winterburn & winter \\
\hline choirmaster & choirboy & choirpractise & choir \\
\hline oilcan & oilfield & oilrig & oil \\
\hline rosebud & rosehip & rosewine & rose \\
\hline bathtub & bathsoap & bathwater & bath \\
\hline bagpipe & baglady & bagmaker & bag \\
\hline fishbowl & fishnet & fishsticks & fish \\
\hline fingernail & fingertip & fingerfed & finger \\
\hline fencepost & fencewall & fenceline & fence \\
\hline needlework & needlecraft & needlepoint & needle \\
\hline anthill & anthive & antcolony & ant \\
\hline coffeepot & coffeemaker & coffeegrounds & coffee \\
\hline coalmine & coalbed & coalengine & coal \\
\hline woodpile & woodblock & woodstove & wood \\
\hline mothball & mothlight & mothwing & moth \\
\hline corkscrew & corkbin & corkwood & cork \\
\hline clamshell & clambed & clamdigger & clam \\
\hline housecoat & housepet & houseparty & house \\
\hline schoolyard & schoolteacher & schoolage & school \\
\hline
\end{tabular}




\section{Appendix B}

Table 7 Stimuli used in Experiment 2

\begin{tabular}{|c|c|c|c|}
\hline Target & Same & Different & $\begin{array}{l}\text { 1st Constituent } \\
\text { Only }\end{array}$ \\
\hline tinfoil & tinbucket & tinsmith & tin \\
\hline oilcan & oilpump & oilstove & oil \\
\hline rosepetal & roseleaf & rosewater & rose \\
\hline earmuff & eardrops & earhole & ear \\
\hline newspaper & newsletter & newsroom & news \\
\hline stonemason & stonecutter & stonework & stone \\
\hline footbath & footstool & footprint & foot \\
\hline milkchurn & milkbucket & milkfat & milk \\
\hline notebook & notecase & notetaker & note \\
\hline toothpaste & toothscraper & toothache & tooth \\
\hline waterbird & waterbeetle & waterjug & water \\
\hline handluggage & handgun & handbrake & hand \\
\hline potholder & pothook & pothole & pot \\
\hline doghouse & dogcollar & dogbite & $\operatorname{dog}$ \\
\hline housecoat & houseplant & houseboat & house \\
\hline doorknob & doorbell & doorkeeper & door \\
\hline worktable & workbench & workload & work \\
\hline seaweed & seawater & seaboat & sea \\
\hline gunpowder & guncase & gunbarrel & gun \\
\hline eyelash & eyelid & eyeglass & eye \\
\hline teabag & teapot & tealeaf & tea \\
\hline lampshade & lamppost & lamplight & lamp \\
\hline fingernail & fingerjoint & fingerfood & finger \\
\hline grapeseed & grapeskin & grapesugar & grape \\
\hline fishbowl & fishknife & fishstew & fish \\
\hline bookcase & bookmarker & bookcollection & book \\
\hline sailboat & sailplane & sailcloth & sail \\
\hline eggcup & eggbeater & eggshell & egg \\
\hline sandstone & sandcastle & sandbox & sand \\
\hline woodpile & woodblock & woodstove & wood \\
\hline windowpane & windowledge & windowdressing & window \\
\hline firewood & firegrate & fireglow & fire \\
\hline breadboard & breadbasket & breadcrumb & bread \\
\hline sunlight & sunbeam & sundeck & sun \\
\hline raincoat & raingauge & rainstorm & rain \\
\hline snowball & snowman & snowplough & snow \\
\hline horseback & horsehair & horseshoe & horse \\
\hline haircream & hairpin & hairfiber & hair \\
\hline paintbrush & paintbox & paintwork & paint \\
\hline shoestring & shoebox & shoemaker & shoe \\
\hline wineglass & wineflask & winestain & wine \\
\hline spacesuit & spaceship & spacetravel & space \\
\hline
\end{tabular}

\section{References}

Baayen, R. H. (2004). Statistics in psycholinguistics: A critique of some current gold standards. In G. Libben \& K. Nault (Eds.), Mental lexical working papers $I$ (pp. 1-45). Edmonton, AB: University of Alberta, Department of Linguistics.

Baayen, R. H. (2007). LanguageR: Data sets and functions with "Analyzing linguistic data: a practical introduction to statistics" (R Package Version 0.1). Edmonton, AB: Author.

Baayen, R. H. (2008). Analyzing linguistic data: A practical introduction to statistics using $R$. Cambridge: Cambridge University Press.

Baayen, R. H., Davidson, D. J., \& Bates, D. M. (2008). Mixed-effects modeling with crossed random effects for subjects and items. Journal of Memory and Language, 59, 390-412.

Bates, D. M. (2005). Fitting linear mixed models in R. R News, 5, 27-30.

Devereux, B., Maguire, P., Costello, F., \& Cater, A. (2006). An analysis of the CARIN model of conceptual combination. In R. Sun \& N. Miyake (Eds.), Proceedings of the 28th annual conference of the cognitive science society (pp. 1222-1227). Mahwah, NJ: Erlbaum.

Downing, P. (1977). On the creation and use of English compound nouns. Language, 53, 810-842.

Estes, Z. (2003). Attributive and relational processes in nominal combination. Journal of Memory and Language, 48, 304-319.

Estes, Z., \& Jones, L. (2006). Priming via relational similarity: A COPPER HORSE is faster when seen through a GLASS EYE. Journal of Memory and Language, 55, 89-101.

Gagné, C. L. (2001). Relation and lexical priming during the interpretation of noun-noun combinations. Journal of Experimental Psychology. Learning, Memory, and Cognition, 27, 236-254.

Gagné, C. L. (2002). Lexical and relational influences on the processing of novel compounds. Brain and Language, 81, $723-735$.

Gagné, C. L. (2009). Psycholinguistic perspectives. In R. Lieber \& P. Stekauer (Eds.), The Oxford handbook of compounding (pp. 255271). Oxford: Oxford University Press.

Gagné, C. L., \& Shoben, E. J. (1997). The influence of thematic relations on the comprehension of modifier-noun combinations. Journal of Experimental Psychology. Learning, Memory, and Cognition, 23, 71-87.

Gagné, C. L., \& Shoben, E. J. (2002). Priming relations in ambiguous noun-noun combinations. Memory \& Cognition, 30, 637-646.

Gagné, C. L., \& Spalding, T. L. (2004). Effect of relation availability on the interpretation and access of familiar noun-noun compounds. Brain and Language, 90, 478-486.

Gagné, C. L., \& Spalding, T. L. (2006). Conceptual combination: Implications for the mental lexicon. In G. Libben \& G. Jarema (Eds.), The representation and processing of compound words (pp. 145-168). Oxford: Oxford University Press.

Gagné, C. L., \& Spalding, T. L. (2009). Constituent integration during the processing of compound words: Does it involve the use of relational structures? Journal of Memory and Language, 60, 20-35.

Gagné, C. L., Spalding, T. L., \& Gorrie, M. (2005a). Sentential context and the interpretation of familiar open-compounds and novel modifier-noun phrases. Language and Speech, 48, 203-221.

Gagné, C. L., Spalding, T. L., \& Ji, H. (2005b). Re-examining evidence for the use of independent relational representations during conceptual combination. Journal of Memory and Language, 53, 445-455.

Gagné, C. L., Spalding, T. L., Figueredo, L., \& Mullaly, A. (2009). Does snowman prime plastic snow? The effect of constituent 
position in using relational information during the interpretation of modifier-noun phrases. Mental Lexicon, 4, 41-76.

Gerrig, R. J. (1989). The time-course of sense creation. Memory \& Cognition, 17, 194-207.

Jones, L. L., Estes, Z., \& Marsh, R. L. (2008). An asymmetric effect of relational integration on recognition memory. The Quarterly Journal of Experimental Psychology, 61, 1169-1176.

Juhasz, B. J., Inhoff, A. W., \& Rayner, K. (2005). The role of interword spaces in the processing of English compound words. Language and Cognitive Processes, 20, 291-316.

Landauer, T. K. (2002). On the computational basis of learning and cognition: Arguments from LSA. In B. H. Ross (Ed.), The psychology of learning and motivation (Vol. 41, pp. 43-84). San Diego, CA: Academic Press.

Landauer, T. K., \& Dumais, S. T. (1997). A solution to Plato's problem: The latent semantic analysis theory of acquisition, induction, and representation of knowledge. Psychological Review, 104, 211-240.

Levi, J. N. (1978). The syntax and semantics of complex nominals. New York: Academic Press.

Libben, G. (1998). Semantic transparency in the processing of compounds: Consequences for representation, processing, and impairment. Brain and Language, 61, 30-44.

Libben, G., Gibson, M., Yoon, Y. B., \& Sandra, D. (2003). Compound fracture: The role of semantic transparency and morphological headedness. Brain and Language, 84, 50-64. doi:10.1016/ S0093-934X(02)00520-5.

Maguire, P., \& Cater, A. (2004, July). Is conceptual combination influenced by word order? Paper presented at the 42nd Annual Meeting of the Association for Computational Linguistics, Barcelona, Spain.

Maguire, P., Devereux, B., Costello, F., \& Cater, A. (2007). A reanalysis of the CARIN theory of conceptual combination. Journal of Experimental Psychology. Learning, Memory, and Cognition, 33, 811-821.

Marslen-Wilson, W., Tyler, L. K., Waksler, R., \& Older, L. (1994). Morphology and meaning in the English mental lexicon. Psychological Review, 101, 3-33.

Murphy, G. L. (1988). Comprehending complex concepts. Cognitive Science, 12, 529-562.
Murphy, G. L. (1990). Noun phrase interpretation and conceptual combination. Journal of Memory and Language, 29, 259-288.

Myers, J. (2007). Generative morphology as psycholinguistics. In G. Jarema \& G. Libben (Eds.), The mental lexicon: Core perspectives (pp. 105-128). Burlington, MA: Elsevier Science.

Pelletier, F. J. (2003). Context dependence and compositionality. Mind and Language, 18, 148-161.

Pinheiro, J. C., \& Bates, D. M. (2000). Mixed-effects models in $S$ and $S$-PLUS. New York, NY: Springer.

Ramey, C. H. (2005). Thematic typicality in modifier-noun conceptual combinations: Controlling for presentation-order effects. Dissertation Abstracts International: Section B. Sciences and Engineering, 66(5b).

Schreuder, R., \& Baayen, R. H. (1995). Modeling morphological processing. In L. B. E. Feldman (Ed.), Morphological aspects of language processing (pp. 131-154). Hillsdale, NJ: Erlbaum.

Schreuder, R., \& Baayen, R. H. (1997). How complex simple words can be. Journal of Memory and Language, 37, 118-139.

Spalding, T. L., \& Gagné, C. L. (2007). Semantic property activation during the interpretation of combined concepts. Mental Lexicon, 2, 25-47.

Spalding, T. L., \& Gagné, C. L. (2008). CARIN theory reanalysis reanalyzed: A comment on Maguire, Devereux, Costello, and Cater (2007). Journal of Experimental Psychology. Learning, Memory, and Cognition, 34, 1573-1578.

Spalding, T. L., Gagné, C. L., Mullaly, A. C., \& Ji, H. (2010). Relation-based interpretations of noun-noun phrases: A new theoretical approach. Linguistische Berichte Sonderheft, 17, 283 315.

Storms, G., \& Wisniewski, E. J. (2005). Does the order of head noun and modifier explain response times in conceptual combination? Memory \& Cognition, 33, 852-861.

Warren, B. (1978). Semantic patterns of noun-noun compounds (Acta Universitatis Gothoburgensis). Goteborg, Sweden: University of Goteborg.

Wisniewski, E. J. (1997). When concepts combine. Psychonomic Bulletin \& Review, 4, 167-183.

Zwitserlood, P. (1994). The role of semantic transparency in the processing and representation of Dutch compounds. Language and Cognitive Processes, 9, 341-368. 